\title{
Characterization of Fuzzy Lattices on a Group with Respect to T-Norms
}

\author{
Dr. M. Marudai \\ Associate Professor \\ PG \& Research Dept. of Mathematics \\ Bharathidasan University \\ Tiruchirappalli - 24 .
}

\author{
V. Rajendran \\ Research Scholar \\ PG \& Research Dept. of Mathematics \\ Bharathidasan University \\ Tiruchirappalli - 24 .
}

\begin{abstract}
We introduce the notion of fuzzy lattice of groups and investigated some of its basic properties. We also study the homomorphic image, pre-image of fuzzy lattices, arbitrary family of fuzzy lattices and lattice fuzzy normal groups using T-norms. we introduce the notion of sensible fuzzy lattice in groups and some related properties of lattices are discussed.
\end{abstract}

\section{AMS Mathematics subject \\ classification (2000) : 06D 72, 06F15, \\ 08A72}

Keywords: Lattice ordered group, Fuzzy lattice, Sensible fuzzy normal lattice, pre-image, direct product.

\section{Introduction}

In the trajectory of stupendous growth of fuzzy set theory, fuzzy algebra has become an important are of research. A. Rosenfeld 1971 [10] used the concept of fuzzy set theory due to Zadeh 1965 [15]. Since then the study of fuzzy algebraic sub structures are important when viewed from a Lattice theoretic point of view. N. Ajmal and K.V. Thomas [1] initiated such types of study in the year 1994. It was latter independently established by N. Ajmal [1] that the set of all fuzzy normal subgroups of a group constitute a sub lattice of the lattice of all fuzzy sub groups of a given group and is Modular. Nanda[4] proposed the notion of fuzzy lattice using the concept of fuzzy partial ordering. More recently in the notion of set product is discussed in details and in the lattice theoretical aspects of fuzzy sub groups and fuzzy normal sub groups are explored. G.S.V. Satya Saibaba [14] initiate the study of L-fuzzy lattice ordered groups and introducing the notice of L-fuzzy sub 1groups. J.A. Goguen [6] replaced the valuation set [0, 1] by means of a complete lattice in an attempt to make a generalized study of fuzzy set theory by studying L-fuzzy sets. A Solairaju and R. Nagarajan [12] introduced the concept of lattice valued Q-fuzzy sub-modules over near rings with respect to T-norms. In this paper we modify the definition of fuzzy lattice and introduce the notion of fuzzy lattice of groups and investigated some of its basic properties. We study the homomorphic image, pre-image of fuzzy lattices, arbitrary family of fuzzy lattices and lattice fuzzy normal groups. We introduce the notion of sensible fuzzy lattices in groups using T-norms and some related properties of lattices are discussed.

\section{SECTION - 2 PRELIMINARIES}

Definition 2.1: A mapping $\mu: \mathrm{X} \rightarrow$

where $\mathrm{X}$ is an arbitrary non empty set and is called fuzzy set in $\mathrm{X}$.

Definition 2.2 : A Lattice ordered group (LG) is a system $\mathrm{G}=(\mathrm{G},+, \leq)$. Where

(i) $(\mathrm{G},+)$ is a group

(ii) $\quad(\mathrm{G}, \square)$ is a lattice

(iii) $\quad \mathrm{x}+\mathrm{a}+\mathrm{y}=\mathrm{b}+\mathrm{y} \rightarrow \mathrm{G}(\mathrm{x}) \leq \max \{\mathrm{G}(\mathrm{a})$,

G(b) \} for all $\quad x, y, a, b \in G$ 
Definition 2.3: Let $X$ be a fuzzy set and $\mu: X \rightarrow G$ be a Lattice ordered group of $X$, then $\mu$ is called fuzzy Lattice ordered group (FLG) if

(i) $\mu(\mathrm{x}+\mathrm{y}) \geq \min \{\mu(\mathrm{x}), \mu(\mathrm{y})\}$

(ii) $\mu(-\mathrm{x})=\mu(\mathrm{x})$

(iii) $\mu(0)=1 \quad$ for all $\mathrm{x}, \mathrm{y} \in \mathrm{G}$.

Definition 2.4: Let $\mu$ be a fuzzy lattice ordered group of $\mathrm{G}$ and $\mu: \mathrm{X} \rightarrow \mathrm{G}$ is called Fuzzy Lattice if

(FL1) $\quad \mu(\mathrm{x}+\mathrm{y}) \quad \geq \min \{\mu(\mathrm{x}), \mu(\mathrm{y})\}$

(FL2) $\quad \mu(-\mathrm{x}) \quad \geq \mu(\mathrm{x})$

(FL3) $\quad \mu(\mathrm{x} V \mathrm{y}) \geq \min \{\mu(\mathrm{x}), \mu(\mathrm{y})\}$

(FL4) $\quad \mu(\mathrm{x} \Lambda \mathrm{y}) \geq \min \{\mu(\mathrm{x}), \mu(\mathrm{y})\}$ for all $\mathrm{x}, \mathrm{y} \in$ G

Definition 2.5: By a t-norm $T$, we mean a function $\mathrm{T}:[0,1] \mathrm{x}[0,1] \rightarrow[0,1]$ satisfying the following conditions

(T1) $\mathrm{T}(0, \mathrm{X})=0$

(T2) $\mathrm{T}(\mathrm{x}, \mathrm{y}) \leq \mathrm{T}(\mathrm{x}, \mathrm{z})$ if $\mathrm{y} \leq \mathrm{z}$

(T3) $\mathrm{T}(\mathrm{x}, \mathrm{y})=\mathrm{T}(\mathrm{y}, \mathrm{x})$

(T4) $\mathrm{T}(\mathrm{x}, \mathrm{T}(\mathrm{y}, \mathrm{z}))=\mathrm{T}(\mathrm{T}(\mathrm{x}, \mathrm{y}), \mathrm{z}), \quad$ for all $\mathrm{x}, \mathrm{y}, \mathrm{z}$ $\in[0,1]$.

Definition 2.6: Let $\mathrm{T}$ be a t-norm. A fuzzy set $\mathrm{A}$ is said to be sensible under $\mathrm{T}$ if $\mathrm{I}_{\mathrm{m}}(\mathrm{A}) \mathrm{C} \Delta \mathrm{T}$.

Where $\Delta \mathrm{T}=\{\mathrm{T}(\alpha, \alpha)=\alpha \mid \alpha \in[0,1]\}$

Definition 2.7: For any fuzzy set $\mu$ in $\mathrm{G}$ and $\mathrm{t} \in[0,1]$, we define the set $U(\mu: t)=\quad\{x \in G \mid \mu(x) \geq$ t \}wich is called an upper cut off $\mu$ and can be used to the characterization of $\mu$.

Definition 2.8: Let f: $G \rightarrow G^{\prime}$ be a lattice group homomorphism and A be a fuzzy lattice of $G^{\prime}$ then $\operatorname{Af}(x)=(A \circ f)(x)=f^{-1}(A)(x)$.

Definition 2.9: Let $\theta: \mathrm{X} \rightarrow \mathrm{Y}$ be a map. $\mathrm{A}$ and $\mathrm{B}$ are fuzzy lattices in $\mathrm{X}$ and $\mathrm{Y}$ respectively. Then the inverse image of $\mathrm{B}$ under $\theta$ is a fuzzy set defined by $\theta^{-1}(\mathrm{~B}) \quad=\mu_{\theta^{-1}(B)}$ where $\mu_{\theta^{-1}(B)}(\mathrm{x})=\mu_{\mathrm{B}}$ $(\theta(\mathrm{x}))$.
Definition 2.10: Any fuzzy lattice $A$ of $G$ is said to be Normal if there exist $\quad \mathrm{x} \in \mathrm{G}$ such that $\mathrm{A}(\mathrm{x})=1$. Not that if $A$ is normal fuzzy lattice of $G$ then $A(0)=1$ and hence $\mathrm{A}$ is normal iff $\mathrm{A}(0)=1$

Definition 2.11: Let $G$ be a group. Let $A$ is fuzzy lattice of $\mathrm{G}$ then $\mathrm{A}$ is called Fuzzy Normal lattice $(F L N)$ if,$A(x y)=A(y x)$. for all $x, y \in G$;

Definition 2.12: Let $\mu_{\mathrm{A}}$ be a fuzzy set of $\mathrm{G}$. Let $\theta$ : $\mathrm{G}$ $\rightarrow \mathrm{G}^{\prime}$ be a map. Define the map $\mu_{\mathrm{A}}(\theta): \mathrm{G} \rightarrow[0,1]$ by $\mu_{A^{\theta}}(\mathrm{x})=\quad \mu_{\mathrm{A}}(\theta(\mathrm{x}))$

\section{SECTION - 3 PROPERITIES OF FUZZY LATTICE}

Proposition 3.1: Let $G$ and $G^{\prime}$ be two Fuzzy Lattice

ordered groups and $\quad \theta: G \rightarrow G^{\prime}$ be a

homomorphism. If B is a Fuzzy Lattice of $G^{\prime}$ then the pre-image $\theta^{-1}(\mathrm{~B})$ is Fuzzy Lattice of G.

Proof : Assume that B is a Fuzzy Lattice of G. Let x, $\mathrm{y} \in \mathrm{G}$.

(FL1) $\mu_{\theta^{-1}(B)}(\mathrm{x}+\mathrm{y}) \quad=\mu_{\mathrm{B}} \theta(\mathrm{x}+\mathrm{y})$

$=\mu_{\mathrm{B}}(\theta(\mathrm{x})+\theta(\mathrm{y}))$

$\geq \min \left\{\mu_{\mathrm{B}}\left(\theta(\mathrm{x}), \mu_{\mathrm{B}} \theta(\mathrm{y})\right)\right\}$

$\geq \min \left\{\mu_{\theta^{-1}(B)}(\mathrm{x}), \mu_{\theta^{-1}(B)}(\mathrm{y})\right\}$

(FL2) $\mu_{\theta^{-1}(B)}(-\mathrm{x}) \quad=\mu_{\mathrm{B}}(\theta(-\mathrm{x}))$

$\geq \mu_{\mathrm{B}}(\theta(\mathrm{x}))$

$\geq \mu_{\theta^{-1}(B)}(\mathrm{x})$

(FL3) $\mu_{\theta^{-1}(B)}(\mathrm{x} V \mathrm{y}) \quad=\mu_{\mathrm{B}} \theta(\mathrm{x} \mathrm{V} \mathrm{y})$

$=\mu_{\mathrm{B}}(\theta \mathrm{x} \mathrm{V} \theta \mathrm{y})$

$\geq \min \left\{\mu_{\mathrm{B}}(\theta(\mathrm{x})), \mu_{\mathrm{B}}(\theta(\mathrm{y}))\right\}$ 
$\geq \min \left\{\boldsymbol{\mu}_{\theta^{-1}(B)}(\mathrm{x}), \mu_{\theta^{-1}(B)}(\mathrm{y})\right\}$

(FL4) $\mu_{\theta^{-1}(B)}\left(\mathrm{x}^{\wedge} \mathrm{y}\right) \quad=\mu_{\mathrm{B}} \theta\left(\mathrm{x}^{\wedge} \mathrm{y}\right)$

$$
=\mu_{\mathrm{B}}\left(\theta \mathrm{x}^{\wedge} \theta \mathrm{y}\right)
$$

$\geq \min \left\{\mu_{\mathrm{B}}(\theta(\mathrm{x})), \mu_{\mathrm{B}}(\theta(\mathrm{y})\}\right.$

$$
\geq \min \left\{\mu_{\theta^{-1}(B)}(\mathrm{x}), \mu_{\theta^{-1}(B)}(\mathrm{y})\right\}
$$

$\therefore \theta^{-1}(\mathrm{~B})$ is a Fuzzy Lattice of G.

Proposition 3.2 :Let $\theta: G \rightarrow G^{\prime}$ be an epimorphism and $B$ is a Fuzzy set in $G^{\prime}$. If $\theta^{-1}(B)$ is Fuzzy Lattice of $G$ then B is Fuzzy Lattice of $G^{\prime}$.

Proof : Let $\mathrm{x}, \mathrm{y} \in \mathrm{G}$, there exist an element $\mathrm{a}, \mathrm{b} \in \mathrm{G}^{\prime}$ such that $\theta(a)=x, \quad \theta(b)=y$

$(\mathrm{FL} 1) \mu_{\mathrm{B}} \quad(\mathrm{x}+\mathrm{y})=\mu_{\mathrm{B}}(\theta(\mathrm{a})+\theta(\mathrm{b}))$

$$
\begin{aligned}
& =\mu_{\mathrm{B}} \theta(\mathrm{a}+\mathrm{b}) \\
& =\mu_{\theta^{-1}(B)}(\mathrm{a}+\mathrm{b})
\end{aligned}
$$

$$
\begin{aligned}
& \geq \min \left\{\mu_{\theta^{-1}(B)}(\mathrm{a}), \mu_{\theta^{-1}(B)}(\mathrm{b})\right\} \\
& \geq \min \left\{\mu_{\mathrm{B}} \theta(\mathrm{a}), \mu_{\mathrm{B}} \theta(\mathrm{b})\right\} \\
& \geq \min \left\{\mu_{\mathrm{B}}(\mathrm{x}), \mu_{\mathrm{B}}(\mathrm{y})\right\}
\end{aligned}
$$

$$
\begin{aligned}
(\mathrm{FL} 2) \mu_{\mathrm{B}}(-\mathrm{x}) \quad & \mu_{\mathrm{B}}(-\theta(\mathrm{a})) \\
& =\mu_{\mathrm{B}}(\theta(-\mathrm{a})) \\
& =\mu_{\theta^{-1}(B)}(-\mathrm{a}) \\
& \geq \mu_{\theta^{-1}(B)}(\mathrm{a}) \\
& \geq \mu_{(B)} \theta(\mathrm{a}) \\
& \geq \mu_{(B)}(\mathrm{x}) \\
(\mathrm{FL} 3) \mu_{\mathrm{B}}(\mathrm{x} \mathrm{V} \mathrm{y})=\mu_{\mathrm{B}}(\theta(\mathrm{a}) & \mathrm{V} \theta(\mathrm{b})) \\
& =\mu_{\mathrm{B}} \theta(\mathrm{a} \mathrm{V} \mathrm{b}) \\
& =\mu_{\theta^{-1}(B)}(\mathrm{a} \mathrm{V} \mathrm{b})
\end{aligned}
$$

$\geq \min \left\{\mu_{\theta^{-1}(B)}(\mathrm{a}), \mu_{\theta^{-1}(B)}(\mathrm{b})\right\}$ $\geq \min \left\{\mu_{\mathrm{B}} \theta(\mathrm{a}), \mu_{\mathrm{B}} \theta(\mathrm{b})\right\}$

$\geq \min \left\{\mu_{\mathrm{B}}(\mathrm{x}), \mu_{\mathrm{B}}(\mathrm{y})\right\}$

$(\mathrm{FL} 4) \mu_{\mathrm{B}}(\mathrm{x} \Lambda \mathrm{y}) \quad=\mu_{\mathrm{B}}(\theta(\mathrm{a}) \Lambda \theta(\mathrm{b}))$

$$
\begin{aligned}
& =\mu_{\mathrm{B}}(\theta(\mathrm{a} \Lambda \mathrm{b})) \\
& =\mu_{\theta^{-1}(B)}(\mathrm{a} \Lambda \mathrm{b})
\end{aligned}
$$

$$
\begin{aligned}
& \geq \min \left\{\mu_{\theta^{-1}(B)}(\mathrm{a}), \mu_{\theta^{-1}(B)}(\mathrm{b})\right\} \\
& \geq \min \left\{\mu_{\mathrm{B}} \theta(\mathrm{a}), \mu_{\mathrm{B}} \theta(\mathrm{b})\right\} \\
& \geq \min \left\{\mu_{\mathrm{B}}(\mathrm{x}), \mu_{\mathrm{B}}(\mathrm{y})\right\}
\end{aligned}
$$

$\therefore \mathrm{B}$ is a Fuzzy Lattice of $\mathrm{G}^{\prime}$.

Proposition 3.3 :If $\left\{A_{i}\right\}$ is a family of Fuzzy Lattice of $G$ then $\cap A_{i}$ is a Fuzzy Lattice of $G$.

Where $\cap \mathrm{A}_{\mathrm{I}}=\left\{\mathrm{x}, \Lambda \mu_{A_{i}}(\mathrm{x}) / \mathrm{x} \in \mathrm{G}\right\}, \quad \mathrm{i} \in \mathrm{A}$.

Proof : Let $x, y \in G$ then for $i \in A$ it follows that

$$
\begin{aligned}
& (\mathrm{FL} 1)\left(\cap \mu_{A_{i}}\right)(\mathrm{x}+\mathrm{y}) \quad=\Lambda \mu_{A_{i}}(\mathrm{x}+\mathrm{y}) \\
& \geq \Lambda \min \left\{\mu_{A_{i}}(\mathrm{x}), \mu_{A_{i}}(\mathrm{y})\right\} \\
& \geq \Lambda \min \left\{\left(\cap \mu_{A_{i}}\right)(\mathrm{x}),\left(\cap \mu_{A_{i}}\right)(\mathrm{y})\right\} \\
& (\mathrm{FL} 2)\left(\cap \mu_{A_{i}}\right)(-\mathrm{x}) \quad=\Lambda \mu_{A_{i}}(-\mathrm{x}) \\
& \geq \Lambda \mu_{A_{i}}(\mathrm{x}) \\
& \geq\left(\cap \mu_{A_{i}}\right)(\mathrm{x}) \\
& \text { (FL3) }\left(\cap \mu_{A_{i}}\right)(\mathrm{x} \mathrm{V} \mathrm{y}) \quad=\Lambda \mu_{A_{i}}(\mathrm{x} \vee \mathrm{y}) \\
& \geq \Lambda \min \left\{\left(\cap \mu_{A_{i}}\right)(\mathrm{x}),\left(\cap \mu_{A_{i}}\right)(\mathrm{y})\right\} \\
& \geq \min \left\{\left(\cap \mu_{A_{i}}\right)(\mathrm{x}),\left(\cap \mu_{A_{i}}\right)(\mathrm{y})\right\} \\
& \text { (FL4) }\left(\cap \mu_{A_{i}}\right)(\mathrm{x} \Lambda \mathrm{y})=\Lambda \mu_{A_{i}}(\mathrm{x} \Lambda \mathrm{y}) \\
& \geq \Lambda \min \left\{\mu_{A_{i}}(\mathrm{x}), \mu_{A_{i}}(\mathrm{y})\right\} \\
& \geq \min \left\{\left(\cap \mu_{A_{i}}\right)(\mathrm{x}),\left(\cap \mu_{A_{i}}\right)(\mathrm{y})\right\} \\
& \therefore \cap A_{i} \text { is a Fuzzy Lattice of } G \text {. }
\end{aligned}
$$


Proposition 3.4 :If A is Fuzzy set in G such that all non empty level subject $U(A ; t)$ is a Fuzzy Lattice of $\mathrm{G}$ then A is Fuzzy Lattice of $\mathrm{G}$.

Proof : Let $x, y \in U(A ; t)$, we have $A(x) \geq t$ and $A(y)$ $\geq \mathrm{t}$. So that $\mathrm{x}+\mathrm{y} \in \mathrm{U}(\mathrm{A} ; \mathrm{t})$ we have $A(x+y) \geq t$.

(FL1) $\mathrm{A}(\mathrm{x}+\mathrm{y}) \quad \geq \mathrm{t}$

$\geq \min \{\mathrm{t}, \mathrm{t}\}$

$\geq \min \{\mathrm{A}(\mathrm{x}), \mathrm{A}(\mathrm{y})\}$

(FL2) $\mathrm{A}(-\mathrm{x})$

$$
\geq \mathrm{t}=\mathrm{A}(\mathrm{x})
$$

(FL3) $\mathrm{A}(\mathrm{x}$ V y $) \quad \geq \mathrm{t}$

$$
\geq \min \{\mathrm{t}, \mathrm{t}\}
$$

$$
\geq \min \{\mathrm{A}(\mathrm{x}), \mathrm{A}(\mathrm{y})\}
$$

(FL4) $\mathrm{A}(\mathrm{x} \wedge \mathrm{y}) \quad \geq$

$$
\geq \min \{\mathrm{t}, \mathrm{t}\}
$$

$\geq \min \{\mathrm{A}(\mathrm{x}), \mathrm{B}(\mathrm{y})\} . \mathrm{A}$ is a Fuzzy

Lattice of G.

Proposition 3.5 :Let A be a Fuzzy Lattice of G. Let $\mathrm{A}^{*}$ be a Fuzzy set in $\mathrm{G}$ defined by $\mathrm{A}^{*}(\mathrm{x})=\mathrm{A}(\mathrm{x})+1-$ $A(0)$, for all $x \in G$. then $A^{*}$ is Normal Fuzzy Lattice of $\mathrm{G}$ which contains $\mathrm{A}$.

Proof : For any $\mathrm{x} \in \mathrm{G}, \mathrm{A}^{*}=$ $\mathrm{A}(\mathrm{x})+1-\mathrm{A}(0)$ (FL1) $\mathrm{A}^{*}(\mathrm{x}+\mathrm{y})=$ $A(x+y)+1$ $-\mathrm{A}(0)$

$$
\geq \min \{\mathrm{A}(\mathrm{x}), \mathrm{A}(\mathrm{y})\}+1-\mathrm{A}(0)
$$

$\geq \min \{\mathrm{A}(\mathrm{x})+1-\mathrm{A}(0), \mathrm{A}(\mathrm{y})+1-\mathrm{A}(0)\}$

$\geq \min \left\{\mathrm{A}^{*}(\mathrm{x}), \mathrm{A}^{*}(\mathrm{y})\right\}$

$$
\begin{aligned}
\left(\text { FL2) } \mathrm{A}^{*}(-\mathrm{x})\right. & =\mathrm{A}(-\mathrm{x})+1-\mathrm{A}(0) \\
& \geq \mathrm{A}(\mathrm{x})+1-\mathrm{A}(0) \\
& \geq \mathrm{A}^{*}(\mathrm{x})
\end{aligned}
$$

(FL3) $\mathrm{A}^{*}(\mathrm{x}$ V y $)=\mathrm{A}(\mathrm{x}$ V y $)+1-\mathrm{A}(0)$

$$
\geq \min \{\mathrm{A}(\mathrm{x}), \mathrm{A}(\mathrm{y})\}+1-\mathrm{A}(0)
$$$$
\geq \min \{\mathrm{A}(\mathrm{x})+1-\mathrm{A}(0), \mathrm{A}(\mathrm{y})+1-\mathrm{A}(0)\}
$$

$\geq \min \left\{\mathrm{A}^{*}(\mathrm{x}), \mathrm{A}^{*}(\mathrm{y})\right\}$

(FL4) $\mathrm{A}^{*}(\mathrm{x} \Lambda \mathrm{y})=\mathrm{A}(\mathrm{x} \Lambda \mathrm{y})+1-\mathrm{A}(0)$

$$
\geq \min \{\mathrm{A}(\mathrm{x}), \mathrm{A}(\mathrm{y})\}+1-\mathrm{A}(0)
$$

$\geq \min \{\mathrm{A}(\mathrm{x})+1-\mathrm{A}(0), \mathrm{A}(\mathrm{y})+1-\mathrm{A}(0)\}$

$\geq \min \left\{A^{*}(x), A^{*}(y)\right\} . A^{*}$ is a Fuzzy Lattice of $G$ and A* C A.
Proposition 3.6 :If $A$ is a Fuzzy Lattice of $G$ and $\theta$ is a homomorphism of $G$ then the Fuzzy set $A^{\theta}$ of $G$ given by

$$
\mathrm{A}^{\theta}=\left\{\left\langle\mathrm{x} ; \mu_{A^{\theta}}(\mathrm{x})\right\rangle, \mathrm{x} \in \mathrm{G}\right\} \text { is a }
$$

Fuzzy Lattice of G.

Proof : For any $x, y \in G$, we have

$$
\begin{aligned}
& \text { (FL1) } \mu_{A^{\theta}}(\mathrm{x}+\mathrm{y})=\mu_{\mathrm{A}} \theta(\mathrm{x}+\mathrm{y}) \\
& =\mu_{\mathrm{A}}(\theta(\mathrm{x})+\theta(\mathrm{y})) \\
& \geq \min \left\{\mu_{\mathrm{A}} \theta(\mathrm{x}), \mu_{\mathrm{A}} \theta(\mathrm{y})\right\} \\
& \geq \min \left\{\mu_{A^{\theta}}(\mathrm{x}), \mu_{A^{\theta}}(\mathrm{y})\right\} \\
& \text { (FL2) } \mu_{A^{\theta}}(-\mathrm{x})=\mu_{A^{\theta}}(-\mathrm{x}) \\
& \geq \mu_{A^{\theta}}(\mathrm{x}) \\
& \text { (FL3) } \mu_{A^{\theta}}(\mathrm{x} V \mathrm{y}) \quad=\mu_{\mathrm{A}} \theta(\mathrm{x} \mathrm{V} \mathrm{y}) \\
& =\mu_{\mathrm{A}}(\theta(\mathrm{x}) \mathrm{V} \theta(\mathrm{y})) \\
& \geq \min \left\{\mu_{\mathrm{A}} \theta(\mathrm{x}), \mu_{\mathrm{A}} \theta(\mathrm{y})\right\} \\
& \geq \min \left\{\mu_{A^{\theta}}(\mathrm{x}), \mu_{A^{\theta}}(\mathrm{y})\right\} \\
& \text { (FL4) } \mu_{A^{\theta}}(\mathrm{x} \Lambda \mathrm{y}) \quad=\mu_{A^{\theta}}(\mathrm{x} \Lambda \mathrm{y}) \\
& =\mu_{\mathrm{A}}(\theta(\mathrm{x}) \Lambda \theta(\mathrm{y})) \\
& \geq \min \left\{\mu_{\mathrm{A}} \theta(\mathrm{x}), \mu_{\mathrm{A}} \theta(\mathrm{y})\right\} \\
& \geq \min \left\{\mu_{A^{\theta}}(\mathrm{x}), \mu_{A^{\theta}}(\mathrm{y})\right\} . A^{\theta} \text { is a Fuzzy }
\end{aligned}
$$

Lattice of $\mathrm{G}$.

Proposition 3.7: Let $\mathrm{T}$ be a continuous t-norm and let $\mathrm{f}$ be a homomorphism on $\mathrm{G}$. If $\mu$ is Fuzzy Lattice of $\mathrm{G}$ then $\mu^{\mathrm{f}} \quad$ is Fuzzy Lattice of $\mathrm{f}(\mathrm{G})$.

Proof :

Let $A_{1}=f^{-1}\left(y_{1}\right), A_{2}=f^{-1}\left(y_{2}\right)$ and $A_{12}=f^{-1}$ $\left(y_{1}+y_{2}\right)$. Where $y_{1}+y_{2} \in f(G)$. Consider the set

$$
A_{1}+A_{2}=\left\{x \in G / x=a_{1}+a_{2}\right\}
$$

For some $a_{1} \in A_{1}$ and $a_{2} \in A_{2}$. If $x \in A_{1}+$ $A_{2}$ then $x=x_{1}+x_{2}$. So that, we have $f(x)=f$ $\left(\mathrm{x}_{1}+\mathrm{x}_{2}\right)$

$$
\begin{gathered}
=f\left(x_{1}\right)+f\left(x_{2}\right) \\
=y_{1}+y_{2} \\
\text { So } x \in f^{-1}\left(y_{1}+y_{2}\right)=A_{12} \text {, thus } A_{1}+A_{2} \text { C }
\end{gathered}
$$

$\mathrm{A}_{12}$ 
It follows that

(FL1) $\mu^{\mathrm{f}}\left(\mathrm{y}_{1}+\mathrm{y}_{2}\right)=\sup \left\{\mu(\mathrm{x}) / \quad \mathrm{x} \in \mathrm{f}^{-1}\left(\mathrm{y}_{1}+\right.\right.$ $\left.\left.\mathrm{y}_{2}\right)\right\}$

$$
\begin{aligned}
& =\sup \left\{\mu(\mathrm{x}) / \mathrm{x} \in \mathrm{A}_{12}\right\} \\
& \geq \sup \left\{\mu(\mathrm{x}) / \mathrm{x} \in \mathrm{A}_{1}+\mathrm{A}_{2}\right\}
\end{aligned}
$$

$\geq \sup \left\{\mu\left(\mathrm{x}_{1}+\mathrm{x}_{2}\right) / \mathrm{x}_{1} \in \mathrm{A}_{1}, \mathrm{x}_{2} \in \mathrm{A}_{2}\right\}$

$\geq \sup \left\{\mathrm{T}\left\{\mu\left(\mathrm{x}_{1}\right), \mu\left(\mathrm{x}_{2}\right) / \mathrm{x}_{1} \in \mathrm{A}_{1}, \mathrm{x}_{2} \in \mathrm{A}_{2}\right\}\right\}$

Since $\mathrm{T}$ is continuous for $\in>0$,

We see that if

$\operatorname{Sup}\left\{\mu\left(\mathrm{x}_{1}\right) / \mathrm{x}_{1} \in \mathrm{A}_{1}\right\}+\mathrm{x}_{1} * \leq \delta$ and

$\operatorname{Sup}\left\{\mu\left(\mathrm{x}_{2}\right) / \mathrm{x}_{2} \in \mathrm{A}_{2}\right\}+\mathrm{x}_{2} * \leq \delta$

$\mathrm{T}\left\{\sup \left\{\mu\left(\mathrm{x}_{1}\right) \mathrm{x}_{1} \in \mathrm{A}_{1}\right\}, \operatorname{Sup}\left\{\mu\left(\mathrm{x}_{2}\right) / \mathrm{x}_{2} \in \mathrm{A}_{2}\right\}\right\}+\mathrm{T}$

$\left(\mathrm{x}_{1} *, \mathrm{x}_{2} *\right) \leq \epsilon$

Choose $a_{1} \in A_{1}$ and $a_{2} \in A_{2}$, such that

$\operatorname{Sup}\left\{\mu\left(\mathrm{x}_{1}\right) / \mathrm{x}_{1} \in \mathrm{A}_{1}\right\}+\mu\left(\mathrm{a}_{1}\right) \leq \delta$ and

$\operatorname{Sup}\left\{\mu\left(\mathrm{x}_{2}\right) / \mathrm{x}_{2} \in \mathrm{A}_{2}\right\}+\mu\left(\mathrm{a}_{2}\right) \leq \delta$

Then

$\mathrm{T}\left\{\operatorname{Sup}\left\{\mu\left(\mathrm{x}_{1}\right) / \mathrm{x}_{1} \in \mathrm{A}_{1}\right\}, \operatorname{Sup}\left\{\mu\left(\mathrm{x}_{2}\right) / \mathrm{x}_{2} \in \mathrm{A}_{2}\right\}+\right.$

$\mathrm{T}\left(\mu\left(\mathrm{a}_{1}\right), \mu\left(\mathrm{a}_{2}\right)\right) \leq \in----(2)$

Thus we have

$\mu^{\mathrm{f}}(\mathrm{x}+\mathrm{y}) \geq \operatorname{Sup}\left\{\mathrm{T}\left(\mu\left(\mathrm{x}_{1}\right), \mu\left(\mathrm{x}_{2}\right)\right) / \quad \mathrm{x}_{1} \in \mathrm{A}_{1}, \mathrm{x}_{2}\right.$

$\left.\in \mathrm{A}_{2}\right\}$

$=\mathrm{T}\left\{\operatorname{Sup}\left(\mu\left(\mathrm{x}_{1}\right) / \mathrm{x}_{1} \in \mathrm{A}_{1}\right), \operatorname{Sup}\left(\mu\left(\mathrm{x}_{2}\right) / \mathrm{x}_{2} \in \mathrm{A}_{2}\right)\right\}$

$\geq \mathrm{T}\left\{\mu^{\mathrm{f}}(\mathrm{x}), \mu^{\mathrm{f}}(\mathrm{y})\right\}$

(FL2) $\mu^{\mathrm{f}}(-\mathrm{x}) \geq \mu^{\mathrm{f}}(\mathrm{x})$

Similarly, we can show

(FL3) $\mu^{\mathrm{f}}(\mathrm{x} V \mathrm{y}) \geq \mathrm{T}\left\{\mu^{\mathrm{f}}(\mathrm{x}), \mu^{\mathrm{f}}(\mathrm{y})\right\}$

and

(FL4) $\mu^{\mathrm{f}}(\mathrm{x} \Lambda \mathrm{y}) \geq \mathrm{T}\left\{\mu^{\mathrm{f}}(\mathrm{x}), \mu^{\mathrm{f}}(\mathrm{y})\right\}$

Proposition 3.8: Let $\mathrm{T}$ be a t-norm. Then every sensible Fuzzy Lattice A of G is Fuzzy Lattice of G.

Proof : Assume that A is sensible Fuzzy Lattice of G then we have

$\begin{array}{lr}\text { (FL1) A }(x+y) \geq & T\{A(x), A(y)\} \\ (\text { FL2) A }(-x) & \geq A(x) \\ \text { (FL3) A (x V y) } & \geq \mathrm{T}\{A(x), A(y)\}\end{array}$

(FL4) $\mathrm{A}(\mathrm{x} \Lambda \mathrm{y}) \geq \mathrm{T}\{\mathrm{A}(\mathrm{x}), \mathrm{A}(\mathrm{y})\}, \quad$ for all $\mathrm{x}$, $\mathrm{y} \in \mathrm{G}$.

Since A is sensible, we have

$\min \{\mathrm{A}(\mathrm{x}), \mathrm{A}(\mathrm{y})\}=\mathrm{T}\{\{\max (\mathrm{A}(\mathrm{x}), \quad \mathrm{A}(\mathrm{y})\},\{\max$

(A(x), A (y) \}\}

$$
\leq \mathrm{T}\{\mathrm{A}(\mathrm{x}), \mathrm{A}(\mathrm{y})\},
$$

$\mathrm{T}(\mathrm{A}(\mathrm{x}), \mathrm{A}(\mathrm{y})) \quad=\min \{\mathrm{A}(\mathrm{x}), \mathrm{A}(\mathrm{y})\}$

It follows that

$\mathrm{A}(\mathrm{x}+\mathrm{y}) \geq \mathrm{T}(\mathrm{A}(\mathrm{x}), \mathrm{A}(\mathrm{y}))$

$$
=\min \{\mathrm{A}(\mathrm{x}), \mathrm{A}(\mathrm{y})\}, \quad \text { for }
$$

all $\mathrm{x}, \mathrm{y} \in \mathrm{G}$.

Similarly, we can show (FL2), (FL3) and (FL4) of Definition (2.4).

So A is a Fuzzy Lattice of G.

Proposition:3.9:Anonto homomorphic image of Fuzzy Lattice with sup property is Fuzzy Lattice.

Proof: Let $\mathrm{f}: \mathrm{G} \rightarrow \mathrm{G}^{\prime}$ be an onto homomorphism of $\mathrm{G}$ and let A be Fuzzy Lattice of $G$ with sup property.

$$
\text { Given } x, y \in G \text {, we let } \quad \mathrm{x}_{\mathrm{o}} \in \mathrm{f}^{-}
$$

${ }^{1}\left(\mathrm{x}^{\prime}\right)$ and $\mathrm{y}_{\mathrm{o}} \in \mathrm{f}^{-1}\left(\mathrm{y}^{\prime}\right)$ be such that

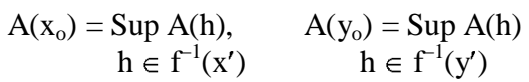

Respectively. Then we can deduce that

$$
\begin{aligned}
& (\text { FL1 }) A^{f}\left(x^{\prime}+y^{\prime}\right)=\sup A(z) \\
& \mathrm{z} \in \mathrm{f}^{-1}\left(\mathrm{x}_{1}+\mathrm{y}_{1}\right) \\
& \geq \operatorname{Min}\left\{\mathrm{A}\left(\mathrm{x}_{\mathrm{o}}\right), \mathrm{A}\left(\mathrm{y}_{\mathrm{o}}\right)\right\} \\
& \geq \operatorname{Min}\{\operatorname{Sup~A(h)} \quad \sup A(h) \\
& \geq \operatorname{Min}\left\{\mathrm{A}^{\mathrm{f}}\left(\mathrm{x}^{\prime}\right), \mathrm{A}^{\mathrm{f}}\left(\mathrm{y}^{\prime}\right)\right\} \\
& A^{f}\left(x^{\prime}+y^{\prime}\right) \geq \operatorname{Min}\left\{A^{f}\left(x^{\prime}\right), A^{f}\left(y^{\prime}\right)\right\} \\
& \text { (FL2) } A^{\mathrm{f}}(-\mathrm{x}) \quad=\operatorname{Sup}\{\mathrm{A}(\mathrm{z}) \\
& \left.\mathrm{z} \in \mathrm{f}^{-1}\left(-\mathrm{x}^{\prime}\right)\right\} \\
& \geq \mathrm{A}\left(\mathrm{y}_{\mathrm{o}}\right) \\
& =\operatorname{Sup} \mathrm{A}(\mathrm{h}) \\
& h \in \mathrm{f}^{-1}\left(\mathrm{y}^{\prime}\right) \\
& =A^{f}\left(y^{\prime}\right)
\end{aligned}
$$


Similarly, we can prove (FL3), (FL4) of Definition

Preposition 3.10: Let $\mathrm{f}: \mathrm{G} \rightarrow \mathrm{G}^{\prime}$ be a Lattice group homomorphism and A be a Fuzzy Lattice of $\mathrm{G}^{\prime}$ then $\mathrm{f}$ ${ }^{1}(\mathrm{~A})$ is a Fuzzy Lattice of G.

Proof : Let $x, y \in G$ and $A$ is a Fuzzy Lattice of $G^{\prime}$.

(FL1) $f^{-1}(A)(x+y)=A f(x+y)$

$$
=A(f(x)+f(y))
$$

$\geq \min \{\mathrm{A}(\mathrm{f}(\mathrm{x})+\mathrm{f}(\mathrm{y})\}$

$\geq \min \left\{\mathrm{f}^{-1}(\mathrm{~A})(\mathrm{x}), \mathrm{f}^{-1}(\mathrm{~A})(\mathrm{y})\right\}$.

$\left(\right.$ FL2) $\mathrm{f}^{-1}(\mathrm{~A})(-\mathrm{x})=\operatorname{A~f}(-\mathrm{x})$

$$
\begin{aligned}
& \geq \mathrm{A} \mathrm{f}(\mathrm{x}) \\
& \geq \mathrm{f}^{-1}(\mathrm{~A})(\mathrm{x}) \\
& (\text { FL3 }) f^{-1}(A)(x \text { V y }) \quad=A f(x \text { V y }) \\
& =\mathrm{A}(\mathrm{f}(\mathrm{x}) \mathrm{V} \mathrm{f}(\mathrm{y})) \\
& \geq \min \{\operatorname{Af}(\mathrm{x}), \operatorname{Af}(\mathrm{y}))\} \\
& \geq \min \left\{\mathrm{f}^{-1}(\mathrm{~A})(\mathrm{x}), \mathrm{f}^{-1}(\mathrm{~A})(\mathrm{y})\right\} \\
& (\mathrm{FL} 4) \mathrm{f}^{-1}(\mathrm{~A})(\mathrm{x} \Lambda \mathrm{y}) \quad=\mathrm{Af}(\mathrm{x} \Lambda \mathrm{y}) \\
& =\mathrm{A}(\mathrm{f}(\mathrm{x}) \Lambda \mathrm{f}(\mathrm{y})) \\
& \geq \min \{\operatorname{Af}(\mathrm{x}), \operatorname{Af}(\mathrm{y}))\} \\
& \geq \min \left\{\mathrm{f}^{-1}(\mathrm{~A})(\mathrm{x}), \mathrm{f}^{1}(\mathrm{~A})(\mathrm{y})\right\}
\end{aligned}
$$

$\therefore \mathrm{f}^{-1}(\mathrm{~A})$ is a Fuzzy Lattice of $\mathrm{G}$.

Proposition 3.11 : Let A be a fuzzy normal lattice of

G. Then for all

$$
x, y \in G, A([x, y])=A(0) \text {. }
$$

Proof : Since A is fuzzy normal lattice of G. We have

$$
\begin{aligned}
& \mathrm{A}(\mathrm{x})=\mathrm{A}\left(\mathrm{y} \mathrm{x} \mathrm{y}^{-1}\right) \text { for all } \mathrm{x}, \mathrm{y} \in \mathrm{G} . \\
& \text { Replacing } \mathrm{x} \text { by } \mathrm{x}^{-1} \text { and } \mathrm{y}^{-1} \mathrm{y}^{-1} \text {, it gives } \\
& \mathrm{A}\left(\mathrm{y}^{-1}\right)=\mathrm{A}\left(\mathrm{x}^{-1} \mathrm{y}^{-1} \mathrm{xy}\right) \text { or } \mathrm{A}\left(\mathrm{x}^{-1} \mathrm{y}^{-1} \mathrm{xyy}^{-1}\right) \\
& =\mathrm{A}\left(\mathrm{y}^{-1}\right) \text { or } \mathrm{A}\left([\mathrm{x}, \mathrm{y}] \mathrm{y}^{-1}\right) \\
& =\mathrm{A}\left(\mathrm{y}^{-1}\right) \text { or } \mathrm{A}[\mathrm{x}, \mathrm{y}] \\
& =\mathrm{A}(0)
\end{aligned}
$$

\section{SECTION - 4 Direct Product of}

\section{Fuzzy Lattices}

Definition 4.1: Let $A_{i}$ be a Fuzzy Lattice of $G_{i}$, for $i=$ $1,2,3 \ldots \mathrm{n}$. Then the product of $\mathrm{A}_{\mathrm{i}}(\mathrm{i}=1,2,3, \ldots \mathrm{n})$ is the function $A_{1} \times A_{2} \times A_{3} \times \ldots \times A_{n}: \quad G_{1} \times G_{2} \times$

$$
\ldots \times G_{n} \rightarrow \text { L defined by }\left(A_{1} \times A_{2} \times A_{3} \times \ldots \times A_{n}\right)
$$$$
\left(\mathrm{x}_{1}, \mathrm{x}_{2}, \ldots \mathrm{x}_{\mathrm{n}}\right)=\min \left\{\mathrm{A}_{1}\left(\mathrm{x}_{1}\right), \mathrm{A}_{2}\left(\mathrm{x}_{2}\right) \ldots \mathrm{A}_{\mathrm{n}}\left(\mathrm{x}_{\mathrm{n}}\right)\right\}
$$

Proposition 4.2: The Direct product of Fuzzy Lattices is a Fuzzy Lattices.

\section{Proof :}

Let $\mathrm{x}=\left(\mathrm{x}_{1}, \mathrm{x}_{2}, \ldots \mathrm{x}_{\mathrm{n}}\right), \mathrm{y}=\left(\mathrm{y}_{1}, \mathrm{y}_{2}, \ldots \mathrm{y}_{\mathrm{n}}\right)$.

$$
\text { Let }\left(x_{1}, x_{2}, \ldots, x_{n}\right),\left(y_{1}, y_{2}, \ldots y_{n}\right) \in A_{1} \times A_{2}
$$

$\times \mathrm{A}_{3} \times \ldots \times \mathrm{A}_{\mathrm{n}}=\lambda$

$(\mathrm{FL} 1) \lambda(\mathrm{x}+\mathrm{y})=\lambda\left(\left(\mathrm{x}_{1}, \mathrm{x}_{2}, \ldots, \mathrm{x}_{\mathrm{n}}\right)+\left(\mathrm{y}_{1}, \mathrm{y}_{2}, \ldots\right.\right.$

$\left.\left.\mathrm{y}_{\mathrm{n}}\right)\right)$

$$
=\mathrm{A}_{1} \times \mathrm{A}_{2} \times \mathrm{A}_{3} \times \ldots \times \mathrm{A}_{\mathrm{n}}\left(\left(\mathrm{x}_{1}+\right.\right.
$$

$\left.\left.\mathrm{y}_{1}\right),\left(\mathrm{x}_{2}+\mathrm{y}_{2}\right) \ldots\left(\mathrm{x}_{\mathrm{n}}+\mathrm{y}_{\mathrm{n}}\right)\right)$

$=\lambda\left(\left(\mathrm{x}_{1}+\mathrm{y}_{1}\right),\left(\mathrm{x}_{2}+\mathrm{y}_{2}\right), \ldots,\left(\mathrm{x}_{\mathrm{n}}+\mathrm{y}_{\mathrm{n}}\right)\right)$

$\geq \min \left\{\mathrm{A}_{1}\left(\mathrm{x}_{1}+\mathrm{y}_{1}\right), \mathrm{A}_{2}\left(\mathrm{x}_{2}+\mathrm{y}_{2}\right), \ldots, \mathrm{A}_{\mathrm{n}}\left(\mathrm{x}_{\mathrm{n}}+\mathrm{y}_{\mathrm{n}}\right)\right\}$

$\geq \min \left\{\min \left\{\mathrm{A}_{1}\left(\mathrm{x}_{1}\right), \mathrm{A}_{1}\left(\mathrm{y}_{1}\right)\right\}, \min \left\{\mathrm{A}_{2}\left(\mathrm{x}_{2}\right), \mathrm{A}_{2}\left(\mathrm{y}_{2}\right)\right\},---\right.$ $\left.\min \left\{A_{n}\left(x_{n}\right), A_{n}\left(y_{n}\right)\right\}\right\}$

$\geq \min \left\{\min \left\{\mathrm{A}_{1}\left(\mathrm{x}_{1}\right), \mathrm{A}_{2}\left(\mathrm{x}_{2}\right), \ldots, \mathrm{A}_{\mathrm{n}}\left(\mathrm{x}_{\mathrm{n}}\right)\right\}\right.$,

$\min \left\{\mathrm{A}_{1}\left(\mathrm{y}_{1}\right), \mathrm{A}_{2}\left(\mathrm{y}_{2}\right) \ldots, \mathrm{A}_{\mathrm{n}}\left(\mathrm{y}_{\mathrm{n}}\right)\right\}$

$\geq \min \left\{\left(\mathrm{A}_{1} \times \mathrm{A}_{2} \times \ldots \times \mathrm{A}_{\mathrm{n}}\right)\left(\mathrm{x}_{1}, \mathrm{x}_{2}, \ldots, \mathrm{x}_{\mathrm{n}}\right)\right.$,

$\left.\left(\mathrm{A}_{1} \times \mathrm{A}_{2} \times \ldots \times \mathrm{A}_{\mathrm{n}}\right)\left(\mathrm{y}_{1}, \mathrm{y}_{2}, \ldots, \mathrm{y}_{\mathrm{n}}\right)\right\}$

$\geq \min \{\lambda(\mathrm{x}), \lambda(\mathrm{y})\}$

(FL2) $\lambda(-\mathrm{x}) \quad=\lambda(-\mathrm{x})$

$=\left(A_{1} \times A_{2} \times \ldots \times A_{n}\right)\left(\left(-x_{1}\right),\left(-x_{2}\right), \ldots,\left(-x_{n}\right)\right)$

$=\min \left\{\mathrm{A}_{1}\left(-\mathrm{x}_{1}\right), \mathrm{A}_{2}\left(-\mathrm{x}_{2}\right), \ldots \mathrm{A}_{\mathrm{n}}\left(-\mathrm{x}_{\mathrm{n}}\right)\right\}$

$\geq \min \left\{A_{1}\left(x_{1}\right), A_{2}\left(x_{2}\right) \ldots A_{n}\left(x_{n}\right)\right\}$

$\geq\left(\mathrm{A}_{1} \times \mathrm{A}_{2} \times \ldots \times \mathrm{A}_{\mathrm{n}}\right)\left(\mathrm{x}_{1}, \mathrm{x}_{2}, \ldots, \mathrm{x}_{\mathrm{n}}\right)$

$\geq \lambda(\mathrm{x})$

(FL3) $\lambda(\mathrm{x} \mathrm{V} \mathrm{y})=\lambda\left(\left(\mathrm{x}_{1}, \mathrm{x}_{2}, \ldots \mathrm{x}_{\mathrm{n}}\right) \mathrm{V}\left(\mathrm{y}_{1}, \mathrm{y}_{2}, \ldots \mathrm{y}_{\mathrm{n}}\right)\right)$

$=\lambda\left(\mathrm{x}_{1} \mathrm{~V} \mathrm{y}_{1}, \mathrm{x}_{2} \mathrm{~V} \mathrm{y}_{2}, \ldots \mathrm{x}_{\mathrm{n}} \mathrm{V} \mathrm{y}_{\mathrm{n}}\right)$

$=\left(A_{1} \times A_{2} \times \ldots \times A_{n}\right)\left(x_{1} V y_{1}, x_{2} V y_{2}, \ldots x_{n} V y_{n}\right)$

$=\min \left\{\mathrm{A}_{1}\left(\mathrm{x}_{1} \mathrm{~V} \mathrm{y}_{1}\right), \mathrm{A}_{2}\left(\mathrm{x}_{\mathrm{x}} \mathrm{V} \mathrm{y}_{2}\right), \ldots, \mathrm{A}_{\mathrm{n}}\left(\mathrm{x}_{\mathrm{n}} \mathrm{V} \mathrm{y}_{\mathrm{n}}\right)\right\}$

$\geq \min \left\{\min \left\{\mathrm{A}_{1}\left(\mathrm{x}_{1}\right), \mathrm{A}_{1}\left(\mathrm{y}_{1}\right)\right\}, \min \left\{\mathrm{A}_{2}\left(\mathrm{x}_{2}\right), \mathrm{A}_{2}\left(\mathrm{y}_{2}\right)\right\}\right.$,

$\left.\ldots, \min \left\{\mathrm{A}_{\mathrm{n}}\left(\mathrm{x}_{\mathrm{n}}\right), \mathrm{A}_{\mathrm{n}}\left(\mathrm{y}_{\mathrm{n}}\right)\right\}\right\}$

$\geq \min \left\{\left\{\min A_{1}\left(x_{1}\right), A_{2}\left(x_{2}\right) \ldots A_{n}\left(x_{n}\right)\right\}, \min A_{1}\left(y_{1}\right)\right.$,

$\left.\left.\mathrm{A}_{2}\left(\mathrm{y}_{2}\right) \ldots \mathrm{A}_{\mathrm{n}}\left(\mathrm{y}_{\mathrm{n}}\right)\right\}\right\}$

$\geq \min \left\{\left(A_{1} \times A_{2} \times \ldots \times A_{n}\right)\left(x_{1}, x_{2}, \ldots, x_{n}\right)\right.$,

$\left.\left(\mathrm{A}_{1} \times \mathrm{A}_{2} \times \ldots \times \mathrm{A}_{\mathrm{n}}\right)\left(\mathrm{y}_{1}, \mathrm{y}_{2}, \ldots, \mathrm{y}_{\mathrm{n}}\right)\right\}$

$\geq \min \{\lambda(\mathrm{x}), \lambda(\mathrm{y})\}$ 
Similarly, we can show (FL4) of definition (2.4).

Proposition 4.3 : The intersection of two Fuzzy Lattices is a Fuzzy Lattice.

Proof :Since A and B are Fuzzy Lattices in G.

$$
\begin{aligned}
& (\mathrm{FL} 1)(\mathrm{A} \cap \mathrm{B})(\mathrm{x}+\mathrm{y}) \quad=\min \{\mathrm{A}(\mathrm{x}+\mathrm{y}), \mathrm{B}(\mathrm{x}+ \\
& \text { y) }\} \\
& \geq \min \{\min \{\mathrm{A}(\mathrm{x}), \mathrm{A}(\mathrm{y})\}\}, \min \{\mathrm{B}(\mathrm{x}), \mathrm{B}(\mathrm{y})\} \\
& \geq \min \{\min \{\mathrm{A}(\mathrm{x}), \mathrm{B}(\mathrm{x})\}, \min \{\mathrm{A}(\mathrm{y}), \mathrm{B}(\mathrm{y})\} \\
& \geq \min \{(\mathrm{A} \cap \mathrm{B})(\mathrm{x}),(\mathrm{A} \cap \mathrm{B})(\mathrm{y})\} \\
& (\mathrm{FL} 2)(\mathrm{A} \cap \mathrm{B})(-\mathrm{x})=\min \{\mathrm{A}(-\mathrm{x}), \mathrm{B}(-\mathrm{x})\} \\
& \geq \min \{\mathrm{A}(\mathrm{x}), \mathrm{B}(\mathrm{x})\} \\
& \geq(\mathrm{A} \cap \mathrm{B})(\mathrm{x})
\end{aligned}
$$

$(F L 3)(A \cap B)(x V y=\min \{A(x V y), B(x V y)\}$

$\geq \min \{\min \{\mathrm{A}(\mathrm{x}), \mathrm{A}(\mathrm{y})\}, \min \{\mathrm{B}(\mathrm{x}), \mathrm{B}(\mathrm{y})\}\}$

$\geq \min \{\min \{\mathrm{A}(\mathrm{x}), \mathrm{B}(\mathrm{x})\}, \min \{\mathrm{A}(\mathrm{y}), \mathrm{B}(\mathrm{y})\}\}$

$\geq \min \{(\mathrm{A} \cap \mathrm{B})(\mathrm{x}),(\mathrm{A} \cap \mathrm{B})(\mathrm{y})\}$

Similarly, we can show (FL4 of Definition (2.4).

Proposition 4.4: If $A$ is a Fuzzy Lattice then $A^{c}$ is also a Fuzzy Lattice.

Proof : For any $\mathrm{x}, \mathrm{y} \in \mathrm{G}$.

$$
\begin{array}{r}
(\text { FL1 }) \begin{array}{r}
A^{c}(x+y) \quad=1-A(x+y) \\
\geq \max \{1-\mathrm{A}(\mathrm{x}), 1-\mathrm{A}(\mathrm{y})\} \\
\geq \max \left\{\mathrm{A}^{\mathrm{c}}(\mathrm{x}), \mathrm{A}^{\mathrm{c}}(\mathrm{y})\right\} \\
=1-\mathrm{A}(-\mathrm{x}) \\
\leq 1-\mathrm{A}(\mathrm{x}) \\
\leq \mathrm{A}^{\mathrm{c}}(\mathrm{x})
\end{array} \\
\begin{array}{r}
\text { (FL2) } \mathrm{A}^{\mathrm{c}}(-\mathrm{x}) \quad=1-\mathrm{A}(\mathrm{x} V \mathrm{y}) \\
\left(\text { FL3) } \mathrm{A}^{\mathrm{c}}(\mathrm{x} \text { V y }) \quad \mathrm{A}(\mathrm{y})\right\} \\
\leq \max \left\{1-\mathrm{A}(\mathrm{x}), 1-\mathrm{A}^{\mathrm{c}}(\mathrm{y})\right\}
\end{array}
\end{array}
$$

Similarly we can show (FL4) of Definition (2.4)

\section{CONCLUSION}

Nandha [4] proposed the concept of Fuzzy Lattice using the notion of Fuzzy partial ordering. But after a critical situation, it has been observed that his definition contains some redundancy. In this paper as a consequence of this observation, we present a modified definition of Fuzzy Lattice and characterization of Fuzzy Lattices.

Applications: Lattice structure has been found to be extremely important in the areas of quantum logic, Erogodic theory, Reynold's operations, Soft Computing, Communication system, Information analysis system, artificial intelligences and physical sciences

ACKNOWLEDGEMENT: The authors are highly grateful to the referees for their valuable comments and suggestions for improving the paper.

The second author also is grateful to Dr.M.Marudai \& Dr.N.Ramanujam, Prof \& Head, Department of Mathematics, Bharathidasan University, Tirchirappalli-24 for valuable suggestions and discussions on this work.

\section{REFERENCES}

[1] N. Ajmal and K.V.Thomas, The Lattice of Fuzzy subgroups and fuzzy normal sub groups, Inform. sci. 76 (1994), 1 - 11.

[2] Birkoff, G : Lattice theory $3^{\text {rd }}$ edn. Amer. Math. Soc. Colloquium pub.25 (1984).

[3] Dubois, D. Prade, H : Fuzzy sets and system - Theory and applications. Academic press. (1980).

[4] Nanda, S : Fuzzy Lattices, Bulletin Calcutta Math. Soc. 81 (1989) $1-2$.

[5] L. Fuchs : Partialy ordered algebraic systems, Pergamon Press, 1963.

[6] J.A. Goguen : L - Fuzzy Sets, J. Math Anal. Appl. 18, 145-174 (1967).

[7] John N Mordeson and D.S. Malik : Fuzzy Commutative Algebra, World Scientific Publishing Co. Pvt. Ltd.

[8] Ath. Kehagia's : The Lattice of fuzzy intervals and sufficient conditions for its distributivity, June 11, 2005.

[9] V. Murali : Lattice of fuzzy algebras and closure system in $\mathrm{I}^{\mathrm{X}}$, Fuzzy sets and systems 41, 101111 (1991). 
[10] A. Rosenfeld : Fuzzy groups, J. Math. Anal. Appl. 35, 512 - 517 (1971).

[11] U.M.Swamy and D. Viswanatha Raju : Algebraic fuzzy systems, Fuzzy sets and system 41, 187-194 (1991).

[12] A. Solairaju and R. Nagarajan : Lattice Valued Q-fuzzy left R - Submodules of Neat Rings with respect to T-Norms, Advances in fuzzy mathematics 4(2), 137 - 145 (2009).
[13] A. Solairaju and R. Nagarajan : A New Structure and Construction of Q-fuzzy groups, Advances in fuzzy mathematics 4(1), 23-29 (2009)

[14] G.S.V. Satya Saibaba. Fuzzy lattice ordered groups, South east Asian Bulletin of Mathematics 32, 749-766 (2008).

[15] L.A. Zadeh : Fuzzy sets, Inform and Control, 8, 338-353 (1965). 